\title{
Reconstrução inguinal com retalho miocutâneo vertical de reto abdominal
}

\author{
Groin reconstruction with vertical rectus abdominis myocutaneous flap
}

\author{
Rosely Moraes \\ GonÇaLves de Moura ${ }^{1}$ \\ Francisco José \\ FonTENele BeZERRA ${ }^{2}$ \\ Janete Clivea Eleuterio \\ DE OliveIrA ${ }^{3}$
}

Trabalho realizado no Instituto do Câncer do Ceará, Fortaleza, CE, Brasil.

Artigo submetido pelo SGP (Sistema de Gestão de Publicações) da RBCP.

Artigo recebido: $23 / 8 / 2010$ Artigo aceito: $26 / 11 / 2010$

\begin{abstract}
RESUMO
Introdução: $O$ carcinoma escamoso é o tumor maligno mais comum de pênis, havendo correlação entre doença local e envolvimento dos linfonodos inguinais por metástase. A linfadenectomia inguinal permite identificar precocemente as micrometástases inguinais, oferecer melhores possibilidades de cura ou tratamento paliativo, porém resulta em grandes perdas de substância. Entretanto, o retalho miocutâneo vertical de reto abdominal (VRAM) permite a cobertura de grandes defeitos de partes moles em áreas como a região inguinal. Método: Foram estudados quatro casos de pacientes com carcinoma escamoso de pênis, submetidos à linfadenectomia e à reconstrução inguinal com VRAM. Resultados: Os pacientes estudados evoluíram sem complicações locais ou de parede abdominal, exceto no caso de reconstrução bilateral, que apresentou áreas de epidermólise e necrose cutânea, com necessidade de enxertia de pele, como também seroma na área receptora. Conclusão: O VRAM permite cobertura de grandes perdas de substância da região inguinal, possibilitando fechamento primário da área doadora.
\end{abstract}

Descritores: Retalhos cirúrgicos. Câncer do pênis. Procedimentos cirúrgicos reconstrutivos. Virilha/cirurgia.

\begin{abstract}
Introduction: Squamous cell carcinoma is the most common malignant tumor of the penis, there was a close correlation between disease location and involvement of inguinal lymph nodes by metastases. Inguinal lymphadenectomy allows early detection of micrometastases inguinal, offer better chances of cure or palliation, but result in large losses of tissue. However the flap, vertical rectus abdominis (VRAM) allows coverage of large soft tissue defects in areas like the groin. Methods: Were studied four cases of patients with squamous cell carcinoma of penis, who underwent inguinal lymphadenectomy and reconstruction with VRAM. Results: The patients evolved without local complications or abdominal wall, except one case of bilateral reconstruction that showed areas of cutaneous necrosis and epidermolysis necessity with skin grafting as well as seroma at the receptor area. Conclusion: The VRAM allows coverage of large losses of substance in the groin, allowing primary closure of the donor area.
\end{abstract}

Keywords: Surgical flaps. Penile neoplasms. Reconstructive surgical procedures. Groin/surgery.

1. Membro Titular da SBCP, Cirurgiã Plástica do Hospital do Câncer do Ceará (ICC); Preceptora do Serviço de Residência Médica em Cirurgia Plástica do Hospital Geral de Fortaleza. Fortaleza, CE, Brasil.

2. Membro Titular da SBCP, Cirurgião Plástico do Hospital do Câncer do Ceará (ICC), Fortaleza, CE, Brasil.

3. Membro Aspirante em Treinamento da Sociedade Brasileira de Cirurgia Plástica (SBCP); Residente de Cirurgia Plástica do Hospital Geral de Fortaleza, Fortaleza, CE, Brasil. 


\section{INTRODUÇÃO}

O câncer de pênis é um tumor raro, com maior incidência a partir dos 50 anos, sendo o carcinoma escamoso (CEP) o mais comum (97\%), podendo estar relacionado a má higiene íntima, fimose e infecção pelo vírus do papiloma humano (HPV) ${ }^{1,2}$.

Algumas características da lesão primária têm sido utilizadas como possíveis fatores indicadores da doença metastática. O grau de diferenciação histológica do tumor é fundamental no envolvimento dos gânglios regionais, havendo progressão do tumor para essas estruturas em até $46 \%$ para tumores moderadamente diferenciados. A profundidade da lesão é outro componente importante, pois independente do grau, 5 a $11 \%$ apresentam progressão para gânglios ${ }^{1-3}$. Porém, as embolizações venosas e linfáticas são apontadas como o principal fator de risco no $\mathrm{CEP}^{3}$. Assim, a presença de metástases nos linfonodos inguinais é a principal variável capaz de afetar a sobrevivência nesses pacientes, apesar de $10 \%$ a $25 \%$ dos doentes com nódulos linfáticos inguinais impalpáveis apresentarem micrometástases não identificadas à ultrassonografia e à tomografia computadorizada ${ }^{1-3}$.

Dessa forma, a linfadenectomia inguinal bilateral representaria o único procedimento capaz de identificar precocemente as micrometástases inguinais ${ }^{3}$.

Apesar da linfadenectomia precoce representar uma forma de tratamento em 70 a $90 \%$ dos pacientes com linfonodos clinicamente negativos, considerando que cerca de $50 \%$ dos pacientes submetidos à linfadenectomia inguinal têm importantes complicações, como infecção de ferida (26\%), necrose e deiscência da ferida operatória (41\%), linfocele $(21 \%)$ e linfedema $(55 \%)$, trata-se de um procedimento com alta morbidade ${ }^{1-3}$. A despeito disso, na doença avançada, quando o tumor infiltra a uretra, o corpo erétil e o corpo esponjoso peniano, alcançando índices de $60 \%$ a $70 \%$ de envolvimento linfonodal, ou nos casos dos tumores pouco diferenciados com $82 \%$ a $100 \%$ de comprometimento, a linfadenectomia inguinal torna-se necessária, até mesmo como tratamento paliativo em alguns casos, pois resultam em feridas dolorosas e exsudativas ${ }^{1-3}$.

No Brasil, esse tipo de tumor representa $2 \%$ de todos os tipos de câncer que atingem o homem ${ }^{4}$, sendo que o estado de São Paulo concentra o maior índice de casos: $24,26 \%$, seguido do Ceará com 12,87\%, Maranhão, com 10,66\% e Rio de Janeiro, com 9,19\% ${ }^{5}$.

Nessas ressecções inguinais, há grande perda de substância e, na maioria dos casos, o fechamento primário não é permitido. Entretanto, o retalho miocutâneo vertical de reto abdominal (VRAM) permite a cobertura de grandes defeitos de partes moles, em que há exposição de estruturas nobres, como a região inguinal, além de possibilitar o fechamento primário da área doadora.

Este estudo foi realizado com o propósito de avaliar os resultados do emprego do retalho VRAM nas reconstruções inguinais, após grandes ressecções de partes moles em tumores penianos com envolvimento metastático inguinal.

\section{MÉTODO}

Foram incluídos neste estudo prospectivo, três pacientes com CEP, submetidos à linfadenectomia inguinal como parte do tratamento e à reconstrução com VRAM. Um dos pacientes foi submetido à reconstrução bilateral, totalizando quatro reconstruções inguinais com VRAM.

A idade dos pacientes variou de 39 a 66 anos (média de 52,5 anos). Dois casos apresentavam CEP (Figura 1) moderadamente diferenciados e invasivos, com estadiamento à primeira consulta de TxN3Mx. O paciente que apresentava acometimento bilateral foi estadiado como T2N $3 \mathrm{Mx}$, neste doente observou-se trombose em veia ilíaca comum e infiltração uretral. As dimensões das peças cirúrgicas variaram, sendo a maior de $15,0 \times 10,0 \times 8,5 \mathrm{~cm}$ e a menor de $12,5 \times 10,0 \times 5,0 \mathrm{~cm}$ (Figura 2), gerando grandes extensões de perdas de partes moles inguinais (Figuras 3 e 4).

As reconstruções foram realizadas pela mesma equipe cirúrgica, em uma única instituição hospitalar, em pacientes oriundos do Serviço Único de Saúde (SUS).

Todas as reconstruções foram imediatas, com retalho VRAM, sendo um caso ipsilateral, um contralateral ao defeito, e um bilateral, todos pediculados na artéria epigástrica inferior profunda.

\section{Técnica cirúrgica}

O segmento vertical de pele sobre o músculo foi marcado,

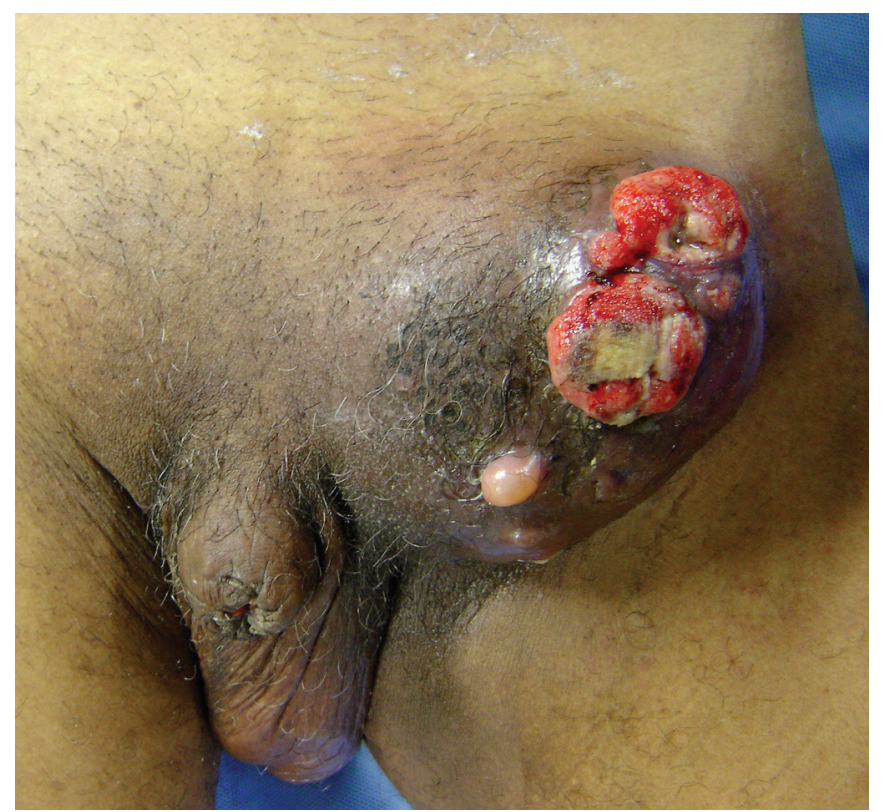

Figura 1 - Invasão tumoral em linfonodo inguinal. 


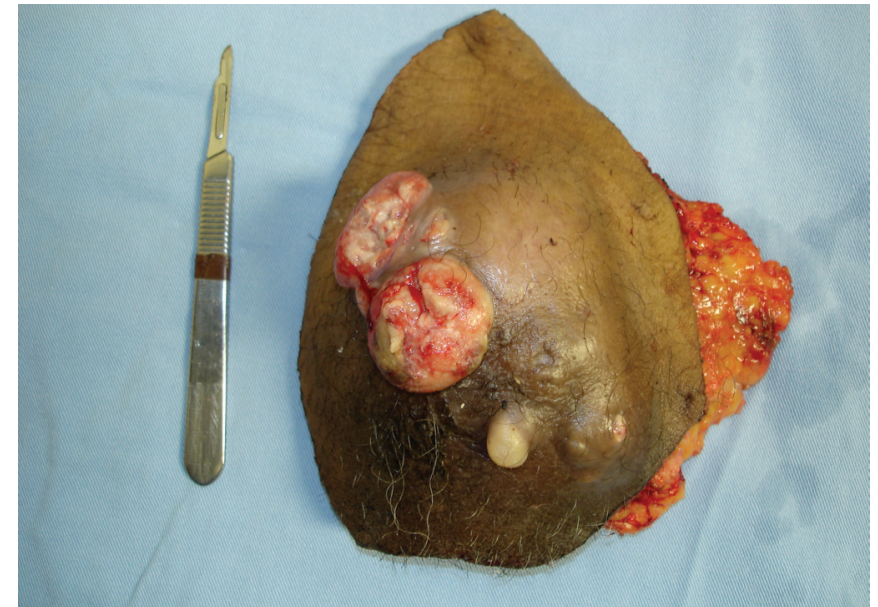

Figura 2 - Peça cirúrgica: produto de linfadenectomia inguinal.

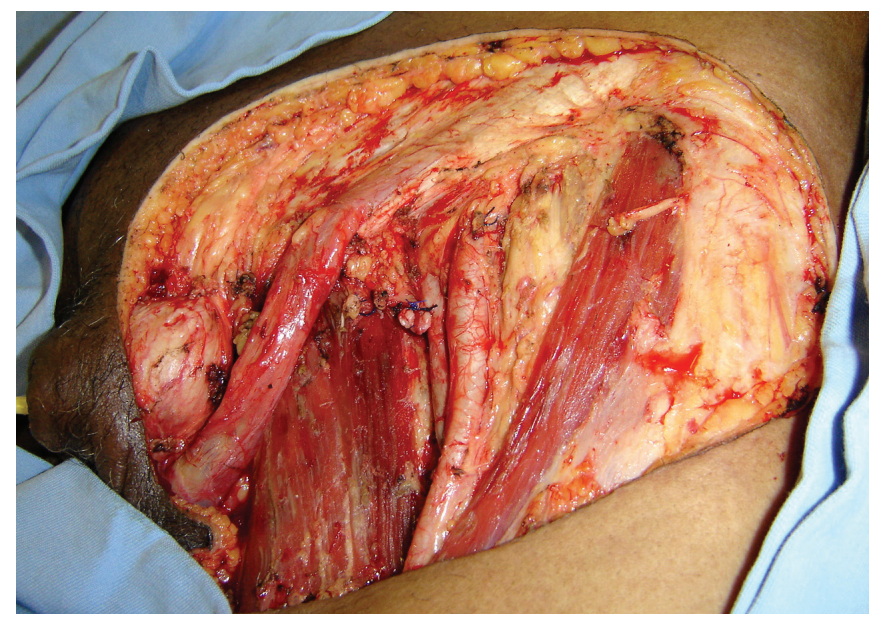

Figura 3 - Área da ressecção do tumor com exposição de vasos inguinais e ligamento inguinal.

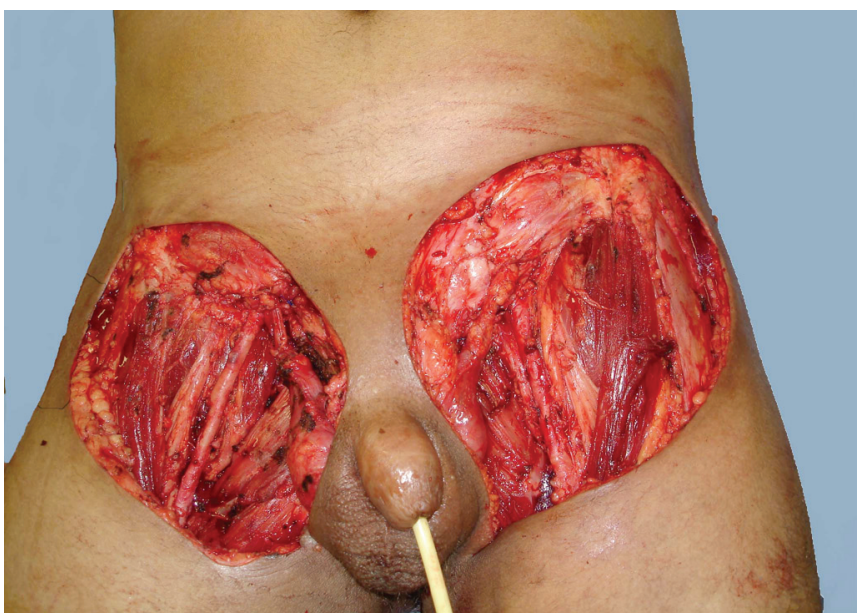

Figura 4 - Extensa perda de substância e sequela de linfadenectomia inguinal bilateral por CEP. estendendo-se desde o local da perda de substância até o rebordo costal, e procedeu-se à elevação do retalho crâniocaudalmente, com secção do músculo reto abdominal e ligadura da artéria epigástrica superior.

Após rotação do retalho, realizou-se o fechamento da parede abdominal com síntese primária das camadas aponeuróticas, aposição de tela sintética não absorvível (polipropileno) inferiormente à linha arqueada, fixação do retalho cutâneo à aponeurose com pontos de adesão, reposicionamento da cicatriz umbilical e síntese por planos da incisão abdominal, representando um fechamento primário em todos os casos.

O VRAM foi posicionado na região inguinal, reparando a perda de substância cutânea em toda sua extensão, oferecendo cobertura das estruturas vasculares expostas (Figuras 5 a 8$)$.

Avaliações locorregionais foram realizadas diariamente, em busca de sinais flogísticos em áreas doadora e receptora ou de sofrimento do retalho.

Os pacientes permaneceram hospitalizados por cerca de $72 \mathrm{~h}$ e foram acompanhados ambulatorialmente.

Esses pacientes receberam radioterapia pós-operatória. Quanto à quimioterapia, dois pacientes foram submetidos ao procedimento no período pós-operatório e um, previamente à cirurgia.

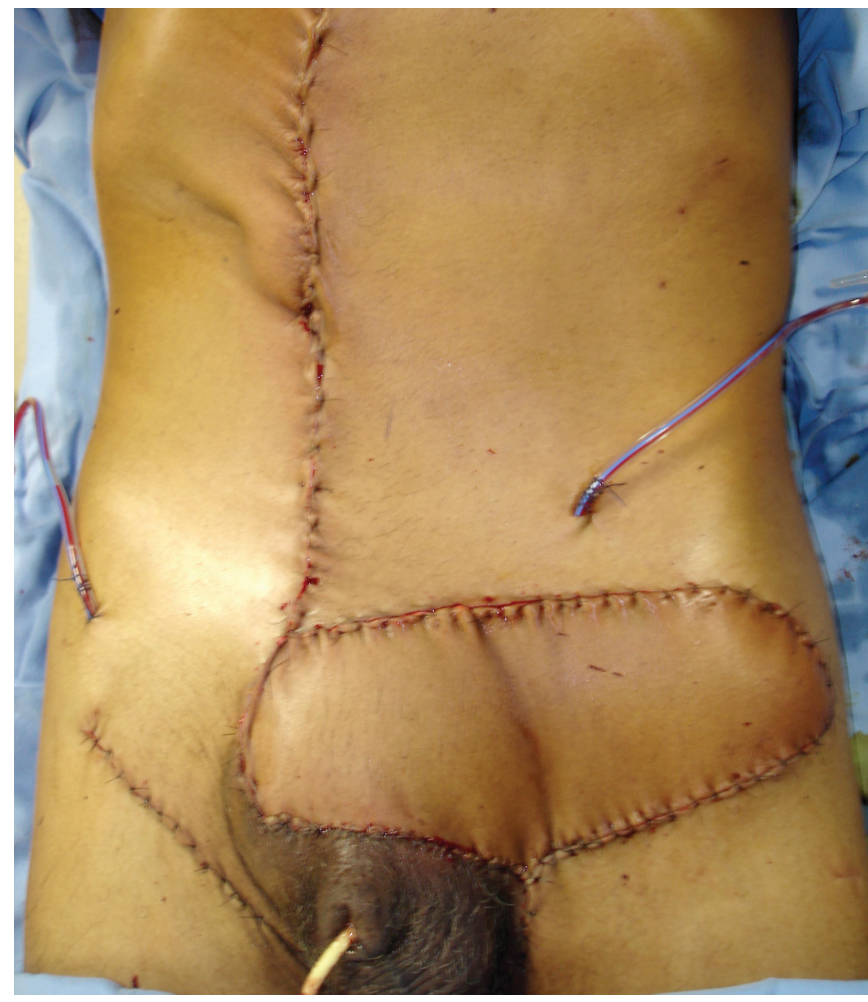

Figura 5 - Aspecto transoperatório: reconstrução inguinal à esquerda com VRAM contralateral. 


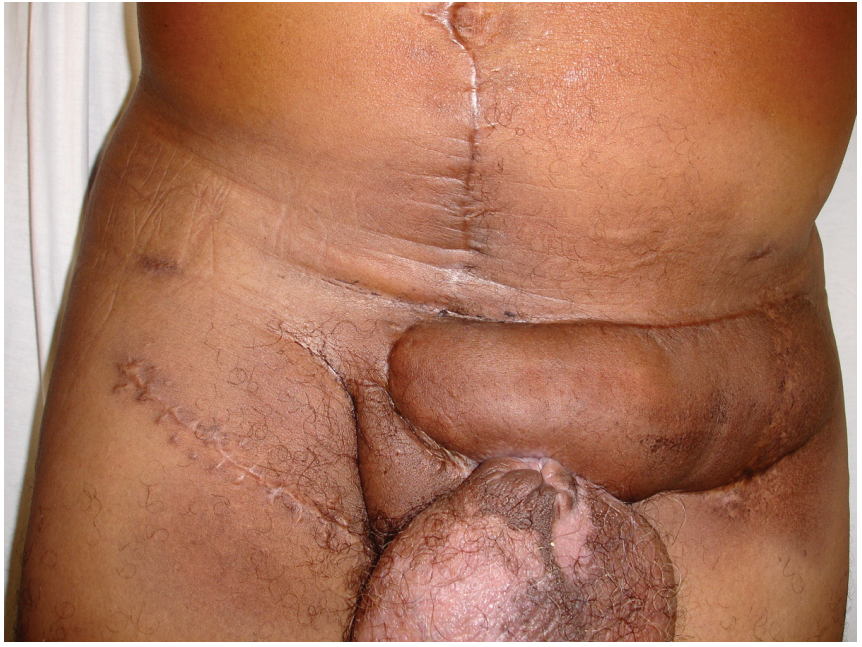

Figura 6 - Pós-operatório tardio de 1 ano.

\section{RESULTADOS}

Os pacientes submetidos à reconstrução inguinal imediata com VRAM evoluíram bem, com melhora significativa da qualidade de vida, realizaram deambulação nas primeiras $24 \mathrm{~h}$ de pós-operatório, reduzindo risco de tromboses.

Apenas o paciente submetido à reconstrução bilateral apresentou no pós-operatório áreas de epidermólise e outras de necrose cutânea pequena, pelo grande edema local em região escrotal, possivelmente devido à dificuldade da drenagem linfática após a ressecção dos linfonodos locais, com necessidade de enxertia de pele, e seroma na área receptora, que foi resolvido com punções.

Os demais pacientes evoluíram sem complicações locais ou de parede abdominal.

\section{DISCUSSÃO}

O músculo reto abdominal, tipo III, segundo Mates e Nahai pediculado nas artérias epigástrica superior e inferior profunda, confere ao retalho versatilidade, por apresentar vascularização segura e amplo arco de rotação, podendo ser utilizado para correção de defeitos da parede torácica, dorso, região inguinal e períneo ${ }^{6}$. Nas reconstruções inguinais, podem ser utilizados retalhos da $\operatorname{coxa}^{7}$, entretanto o VRAM fornece a cobertura de grandes defeitos de partes moles em que há exposição de estruturas nobres, como a região inguinal, além de permitir fechamento primário da área doadora ${ }^{8}$.

Nos casos apresentados, havia exposição importante de estruturas nobres, como vasos profundos da região inguinal, ligamento inguinal e parte de testículos, que tinham que ser recobertas com tecido suficientemente espesso para proteção

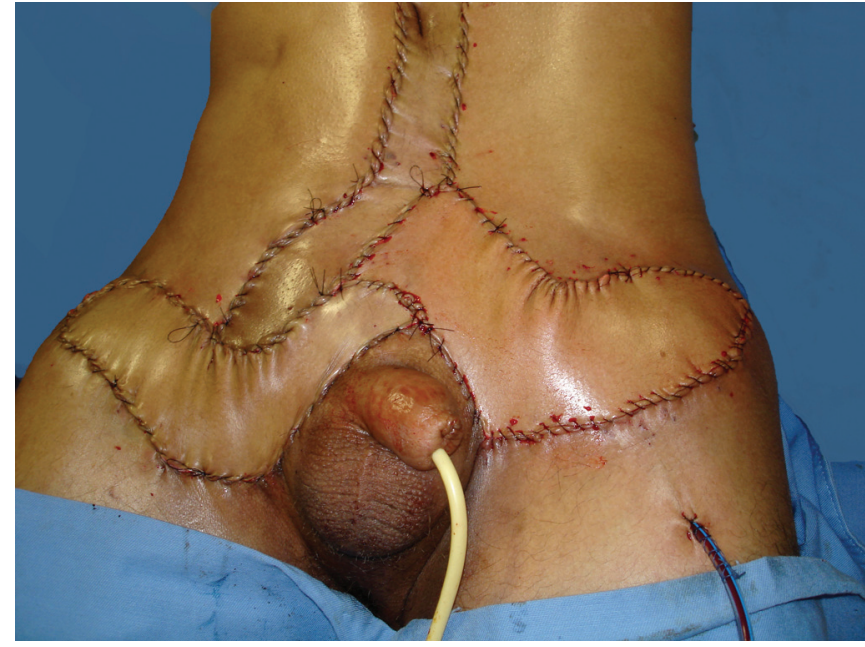

Figura 7 - Aspecto transoperatório: reconstrução inguinal com VRAM bilateral.

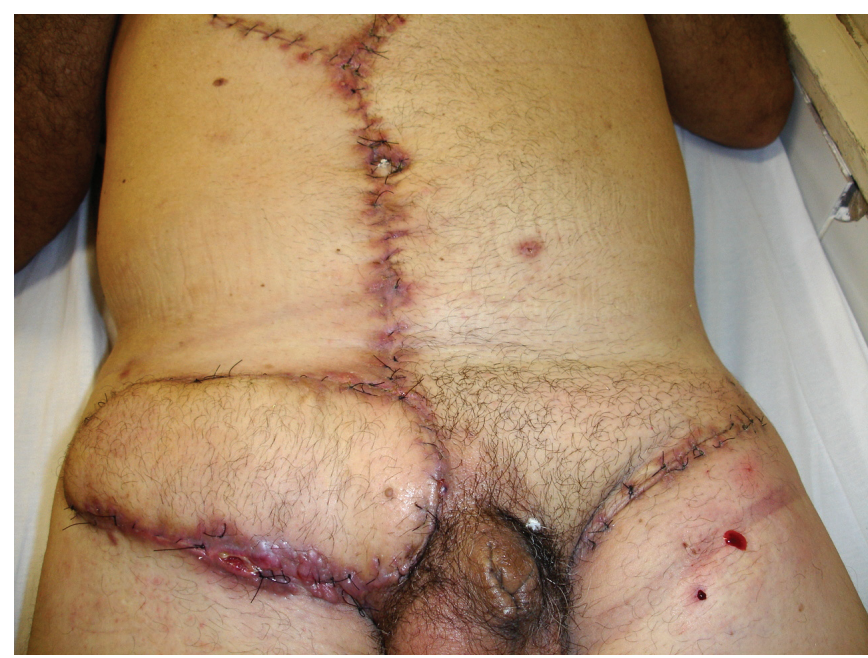

Figura 8-Pós-operatório: 15 dias de reconstrução com VRAM ipsilateral à direita.

das mesmas e com um retalho bem vascularizado e seguro, que tivesse pouco risco de necrose, evitando assim a maior morbidade desses pacientes já espoliados, num segundo procedimento. Acreditamos que o retalho VRAM foi uma boa opção nesses casos, permitindo a cobertura dessa região e fechamento primário da área doadora.

Logan e Mathes ${ }^{9}$ relataram as vantagens do emprego do VRAM pediculado inferiormente na artéria epigástrica profunda, por oferecer quantidade de tecido suficiente para cobertura de grandes defeitos inguinais. Deo et al. ${ }^{10}$ avaliaram os resultados de oito pacientes submetidos à reconstrução de grandes defeitos de partes moles da parede torácica, inguinal, períneo e coxa com VRAM e concluíram que se trata de um 
retalho versátil, confiável e sem complicações como infecção de ferida, necrose do retalho ou hérnia abdominal.

Küntscher et al. ${ }^{11}$ analisaram os resultados do emprego do VRAM para cobertura de defeitos após ressecção de tumor peniano em sete pacientes e concluíram que esse retalho representa importante opção nesses casos. Kayes et al. ${ }^{12}$ estudaram quatro pacientes com doença metastática subcutânea por câncer avançado de pênis, submetidos à ressecção paliativa agressiva e à reconstrução com VRAM e não observaram complicações cirúrgicas. Verificaram, ainda, controle local da doença e melhora da qualidade de vida, assim como nos casos relatados em nosso estudo, nos quais também houve melhora significativa da qualidade de vida após realização de cirurgia higiênica de ressecção dos tumores supurados inguinais, seguida de imediata reconstrução.

Nelson e Butler ${ }^{13}$ relataram menores índices de complicações com o emprego do VRAM em 119 pacientes em comparação ao retalho da coxa, após estudo de pacientes submetidos a ressecção abdominoperineal ou exenteração pélvica, considerando complicações como celulite, abscessos e deiscência de ferida.

Outros retalhos, como o reto femoral, sartório com retalho de pele abdominal, retalho do músculo gracilis, retalho do músculo oblíquo interno, vasto lateral e tensor da fáscia lata, também têm sido descritos ${ }^{6-13}$. No entanto, esses retalhos não são isentos de complicações ou morbidades, a maioria relacionada à morbidade da área doadora ou ao tempo de repouso, nos casos de retalhos da coxa, o que pode ter um risco adicional para pacientes idosos, como trombose venosa profunda ${ }^{14}$.

Não encontramos na literatura relatos recentes do emprego do VRAM bilateral para reconstrução inguinal bilateral.

O retalho VRAM aqui descrito trata-se de um retalho miocutâneo de pedículo inferior do reto abdominal, portanto inverso da descrição original, em que é utilizado pediculado na artéria epigástrica superior ${ }^{15,16}$. O uso do retalho pediculado inferiormente confere maior versatilidade ao retalho, possibilitando reconstruções de defeitos de abdome inferior, região inguinal, perineal e raiz da coxa, sendo uma nova alternativa para essas regiões.

\section{CONCLUSÃO}

Concluímos que o VRAM representa um excelente retalho miocutâneo para cobertura de grandes perdas de substância da região inguinal, com boa espessura, além de permitir fechamento primário tanto do defeito quanto da área doadora, sem aumentar a morbidade, e melhorando a qualidade de vida dos pacientes com tumores malignos de pênis em estádio avançado.

\section{REFERÊNCIAS}

1. Singh I, Khaitan A. Current trends in the management of carcinoma penis: a review. Int Urol Nephrol. 2003;35(2):215-25.

2. Ficarra V, Martignoni G, Maffei N, Cerruto MA, Novara G, Cavalleri $\mathrm{S}$, et al. Predictive pathological factors of lymph nodes involvement in the squamous cell carcinoma of the penis. Int Urol Nephrol. 2002;34(2):245-50.

3. Cologna AJ, Faria SM, Martins ACP, Suaid HJ, Tucci Jr S, Reis RB. Avaliação dos gânglios regionais no carcinoma epidermóide do pênis (CEP) através de um índice de risco. Acta Cir Bras. 2001;16(suppl.1):32-5.

4. Sociedade Brasileira de Urologia. $1^{\circ}$ Estudo epidemiológico sobre o câncer de pênis. Disponível em: www.sbu.org.br. Acesso em: jun. 2010.

5. INCA. Disponível em: www.inca.gov.br . Acesso em: jun. 2010.

6. Lee MJ, Dumanian GA. The oblique rectus abdominis musculocutaneous flap: revisited clinical applications. Plast Reconstr Surg. 2004;114(2):367-73

7. Rifaat MA, Abdel Gawad WS. The use of tensor fascia lata pedicled flap in reconstructing full thickness abdominal wall defects and groin defects following tumor ablation. J Egypt Natl Canc Inst. 2005;17(3):139-48.

8. Parrett BM, Winograd JM, Garfein ES, Lee WP, Hornicek FJ, Austen WG Jr. The vertical and extended rectus abdominis myocutaneous flap for irradiated thigh and groin defects. Plast Reconstr Surg. 2008;122(1):171-7.

9. Logan SE, Mathes SJ. The use of a rectus abdominis myocutaneous flap to reconstruct a groin defect. Br J Plast Surg. 1984;37(3):351-3.

10. Deo SV, Nootan KS, Niranjan B, Dinesh K. Vertical rectus abdominis myocutaneous flap cover for lower abdomen, chest wall, groin and thigh defects following resection of malignant tumours. Indian J Cancer. 2001;38(1):33-7.

11. Küntscher MV, Mansouri S, Noack N, Hartmann B. Versatility of vertical rectus abdominis musculocutaneous flaps. Microsurgery. 2006;26(5):363-9.

12. Kayes OJ, Durrant CA, Ralph D, Floyd D, Withey S, Minhas S. Vertical rectus abdominis flap reconstruction in patients with advanced penile squamous cell carcinoma .BJU Int. 2007;99(1):37-40.

13. Nelson RA, Butler CE. Surgical outcomes of VRAM versus thigh flaps for immediate reconstruction of pelvic and perineal cancer resection defects. Plast Reconstr Surg. 2009;123(1):175-83.

14. Zeng A, Xu J, Yan X, You L, Yang H. Pedicled deep inferior epigastric perforator flap: an alternative method to repair groin and scrotal defects. Ann Plast Surg. 2006;57(3):258-8.

15. Dinner MI, Labandter HP, Dowden RV. The role of the rectus abdominis myocutaneous flap in breast reconstruction. Plast Reconstr Surg. 1982;69(2):209-15.

16. Robbins TH. Post-mastectomy breast reconstruction using a rectus abdominis musculocutaneous island flap. Br J Plast Surg. 1981;34(3):286-90.

\section{Correspondência para: Rosely Moraes Gonçalves de Moura}

Rua Rui Monte, 1220 - Bloco 14 - Apto. 401 - Antonio Bezerra - Fortaleza, CE, Brasil - CEP 60360-640

E-mail: roselymgdemoura@hotmail.com 